\title{
How Many Patients with Type 2 Diabetes Meet the Inclusion Criteria of the Cardiovascular Outcome Trials with SGLT2 Inhibitors? Estimations from a Population Database in a Mediterranean Area
}

\author{
Silvia Canivell, ${ }^{1,2,3}$ Manel Mata-Cases $\left(\mathbb{D},{ }^{1,4,5}\right.$ Bogdan Vlacho, ${ }^{1}$ Mònica Gratacòs, ${ }^{1}$ \\ Jordi Real, ${ }^{1,4}$ Dídac Mauricio $\mathbb{D}^{1,4,6}$ and Josep Franch-Nadal $\oplus^{1,4,7}$ \\ ${ }^{1}$ DAP-Cat Group, Unitat de Suport a la Recerca Barcelona, Fundació Institut Universitari per a la recerca a l'Atenció Primària de \\ Salut Jordi Gol i Gurina (IDIAPJGol), Barcelona, Spain \\ ${ }^{2}$ Primary Health Care Center Sant Martí de Provençals, Gerència d'Atenció Primaria, Institut Català de la Salut, Barcelona, Spain \\ ${ }^{3}$ Health Sciences Research Institute and Hospital Universitari Germans Trias i Pujol, Badalona, Spain \\ ${ }^{4}$ CIBER of Diabetes and Associated Metabolic Diseases (CIBERDEM), Instituto de Salud Carlos III (ISCIII), Barcelona, Spain \\ ${ }^{5}$ Primary Health Care Center La Mina, Gerència d'Àmbit d'Atenció Primària Barcelona Ciutat, Institut Català de la Salut, \\ Sant Adrià de Besòs, Spain \\ ${ }^{6}$ Department of Endocrinology \& Nutrition, Hospital de la Santa Creu i Sant Pau, Autonomous University of Barcelona, \\ Barcelona, Spain \\ ${ }^{7}$ Primary Health Care Center Raval Sud, Gerència d’Atenció Primaria, Institut Català de la Salut, Barcelona, Spain
}

Correspondence should be addressed to Dídac Mauricio; didacmauricio@gmail.com

and Josep Franch-Nadal; josep.franch@gmail.com

Received 18 April 2019; Revised 30 September 2019; Accepted 16 October 2019; Published 11 November 2019

Academic Editor: Kim Connelly

Copyright $\odot 2019$ Silvia Canivell et al. This is an open access article distributed under the Creative Commons Attribution License, which permits unrestricted use, distribution, and reproduction in any medium, provided the original work is properly cited.

Objective. Regulatory agencies require the assessment of cardiovascular (CV) safety for new type 2 diabetes (T2D) therapies through CV outcome trials (CVOTs). However, patients included in CVOTs assessing sodium-glucose cotransporter-2 inhibitors (SGLT2i) might not be representative of those seen in clinical practice. This study examined the proportion of patients that would have been enrolled into three main SGLT2i CVOTs to determine whether these trials' eligibility criteria can be applied to a real-world Mediterranean T2D population. Methods. Cross-sectional, retrospective, cohort study of T2D patients registered in primary care centres of the Catalan Institute of Health using medical records from a population database (SIDIAP) that includes approximately $74 \%$ of the population in Catalonia (Spain). Eligibility criteria were according to those of three SGLT2i CVOTs: EMPA-REG OUTCOME (empagliflozin), CANVAS (canagliflozin), and DECLARE-TIMI 58 (dapagliflozin). Results. By the end of 2016, the database included 373,185 patients with T2D with a mean age of $70 \pm 12$ years, $54.9 \%$ male, with a mean duration of T2D of $9 \pm 6$ years, and a mean glycated haemoglobin (HbA1c) of $7.12 \% \pm 1.32$ (59\% with HbAlc $<7 \%$ ). Of these, 86,534 (23\%) had established CV disease and $28 \%$ chronic renal failure (estimated glomerular filtration $<60 \mathrm{ml} / \mathrm{min} / 1.73 \mathrm{~m}^{2}$ ). Among all included patients, only $8.2 \%$ would have qualified for enrolment into the EMPA-REG OUTCOME trial, $29.6 \%$ into the CANVAS program, and 38\% into the DECLARE-TIMI 58 trial. The main limiting factors for inclusion would have been a previous history of $\mathrm{CV}$ disease and the baseline HbAlc value. Conclusion. The external validity of the analysed CVOTs is clearly limited when applying the same eligibility criteria to a T2D Mediterranean population. 


\section{Introduction}

Patients with type 2 diabetes (T2D) have an increased risk of renal and cardiovascular disease (CVD) and mortality [1]. Therefore, improvement in cardiovascular (CV) health is one of the main goals of diabetes management. While tight good glycaemic control in T2D is associated with reduced risk of microvascular disease [2, 3], the benefit regarding macrovascular disease is less clear [4-6]. Indeed, a meta-analysis combining the results of large-scale trials showed that intensive glucose-lowering therapy was associated with a significant reduction in the overall incidence of $\mathrm{CV}$ events and myocardial infarction compared to conventional therapy (odds ratio (OR) 0.89, $P=0.001$; OR 0.84, $P<0.001$, respectively) [7]. However, there was no difference in the incidence of CV mortality [7]. Both the US Food and Drug Administration (FDA) and the European Medicines Agency (EMA) require, for each new antidiabetic therapy to treat $\mathrm{T} 2 \mathrm{D}$, to show a neutral or beneficial effect in CV safety through the conduction of CV outcome trials (CVOTs) $[8,9]$.

Sodium-glucose cotransporter-2 inhibitors (SGLT2i) are a promising group of new drugs for the treatment of T2D that act by preventing the reabsorption of glucose from the proximal renal tubule in the kidney [10]. Additionally, they have numerous pleiotropic effects such as reducing blood plasma glucose, body weight, and blood pressure and inducing natriuresis [10]. In the particular case of SGLT2i, recent CVOTs have shown renal and CV benefits and further studies are ongoing [11-13]. However, one of the major issues of randomised clinical trials (RCTs) is the external validity of the results, that is, to what extent the overall average effect of the treatment can be generalised to a particular group of patients or clinical setting [14]. For instance, the external validity can be challenged by the trial's setting (e.g., differences between countries regarding the health care system, disease management, or natural history of the disease), the inclusion and exclusion criteria, or differences between the protocol trial and routine clinical practice, among other issues [14].

The results of the CVOTs of three SLGT2 inhibitors available in Spain published to date are EMPA-REG OUTCOME with empagliflozin [15], CANVAS with canagliflozin [16], and DECLARE-TIMI 58 with dapagliflozin [17]. The EMPA-REG OUTCOME trial included only patients with established CV disease (CVD), i.e., secondary prevention [15]. The other two trials included secondary prevention patients and also patients with $\mathrm{CV}$ risk factors who have not yet developed CVD (primary prevention): with $\geq 1 \mathrm{CV}$ risk factors in the DECLARE-TIMI 58 trial [17] and with $\geq 2 \mathrm{CV}$ risk factors in the CANVAS study [16]. Since the eligibility criteria varied among these SGLT2i CVOTs, it was expected that the external validity of the different studies might also differ; thus, the trial population does not actually represent the general T2D population. Indeed, the external validity of CVOTs regarding SGLT2i has been assessed by two recently published studies using clinical routine data from the US and Northern Europe [18, 19]. Both studies found large differences between trials regarding the proportion of patients seen in clinical practice that would have met entry criteria in these CVOTs, with the DECLARE-TIMI 58 trial as the most generalisable and applicable one. Moreover, the results from the study conducted in Northern Europe were consistent across all four included countries (i.e., Germany, The Netherlands, Norway, and Sweden) [18]. However, there is no published information from Southern European countries so far, although the distribution of $\mathrm{CV}$ risk factors as well as the prevalence of CVD in patients with diabetes differs across regions in Europe [20, 21]. Based on these potential differences, we hypothesised that the external validity of the CVOTs could be different when the general T2D population is estimated in a Mediterranean country.

The aim of the present study was to determine the proportion of patients with T2D in primary care that would be eligible for inclusion in the CVOTs of SLGT2i in the population served by the Catalonian Health Institute in Catalonia, a Mediterranean area in the northeast of Spain.

\section{Materials and Methods}

2.1. Design. This was a cross-sectional retrospective study of the T2D population attended at primary care centres of Catalonia, an autonomous region located in the northeast of Spain, corresponding to $12 \%$ of the total Spanish population. We compared the potential eligibility of patients to those included in publications describing three completed SLGT2i CVOTs [15-17].

The study was approved by the Ethics Committee of the Primary Health Care University Research Institute (IDIAP) Jordi Gol in accordance with the Spanish regulations on observational studies. This retrospective study using anonymised data did not require obtaining informed consent from the patients.

2.2. Data Source. Data from patients were extracted from the SIDIAP database, which contains anonymised longitudinal patient information obtained from electronic clinical records; it incorporates available information from 288 primary care teams of the Catalonian Health Institute (ICS), which serves around 5.6 million people, $74 \%$ of the total population in Catalonia. The SIDIAP includes demographic, clinical, and pharmacy-invoicing data provided by the CatSalut general database, and it has already been used for epidemiological research purposes and realworld evidence [22-24].

2.3. Inclusion and Exclusion Criteria. The study population consisted of patients aged 18 years or older with a diagnosis of T2D (International Classification of Diseases (ICD10) codes E11, E11.0-E11.9, E14, and E14.0-E14.9) as of 31 December 2016 (index date). We excluded patients with a diagnosis of type 1 diabetes, gestational diabetes mellitus, and any other type of diabetes. After this initial selection, we applied the inclusion and exclusion criteria from the CVOTs for the SGLT2i commercialised up to date in Spain, namely, empagliflozin (EMPA-REG OUTCOME 
TABLE 1: Clinical characteristics of patients with T2D registered in the SIDIAP database.

\begin{tabular}{|c|c|c|c|}
\hline Characteristics & $\begin{array}{c}\text { All population } \\
(N=373,185)\end{array}$ & $\begin{array}{l}\text { Secondary prevention patients* } \\
\qquad(N=86,534)\end{array}$ & $\begin{array}{l}\text { Primary prevention patients** } \\
\qquad(N=286,651)\end{array}$ \\
\hline Gender, male, $n(\%)$ & $204,707(54.9)$ & $56,882(65.7)$ & $147,825(51.6)$ \\
\hline Age (years), mean (SD) & $70.1(12.3)$ & $74.8(10.5)$ & $68.7(12.5)$ \\
\hline Current smokers, $n(\%)$ & $52,744(14.1)$ & $11,058(12.8)$ & $41,686(14.5)$ \\
\hline \multicolumn{4}{|l|}{ BMI $\left(\mathrm{kg} / \mathrm{m}^{2}\right), n(\%)$} \\
\hline$\geq 30$ & $144,592(44.9)$ & $30,366(40.3)$ & $114,226(46.4)$ \\
\hline$>45$ & $3,905(1.2)$ & $521(0.7)$ & $3,384(1.4)$ \\
\hline $\begin{array}{l}\text { Duration of diabetes (years), } \\
\text { mean (SD) }\end{array}$ & $9.3(6.2)$ & $10.9(6.7)$ & $8.8(6.0)$ \\
\hline $\operatorname{HbAlc}(\%)^{\dagger}$, mean $(\mathrm{SD})$ & $7.12(1.32)$ & $7.16(1.32)$ & $7.10(1.33)$ \\
\hline HbAlc $\leq 7 \%, n(\%)$ & $194,751(59.0)$ & $43,664(56.6)$ & $151,087(59.8)$ \\
\hline Hypertension, $n(\%)$ & $268,394(71.9)$ & $70,026(80.9)$ & $198,368(69.2)$ \\
\hline Dyslipidemia, $n(\%)$ & $223,785(60.0)$ & $56,194(64.9)$ & $167,591(58.5)$ \\
\hline \multicolumn{4}{|l|}{$\operatorname{eGFR}\left(\mathrm{ml} / \mathrm{min} / 1.73 \mathrm{~m}^{2}\right)^{\dagger}, n(\%)$} \\
\hline$\geq 60$ & $241,958(72)$ & $45,598(58)$ & $196,360(76)$ \\
\hline $30-60$ & $80,978(24)$ & $27,651(35)$ & $53,327(21)$ \\
\hline$<30$ & $13,262(4)$ & $5,909(7)$ & $7,353(3)$ \\
\hline UACR $\geq 30 \mathrm{mg} / \mathrm{g}, n(\%)$ & $51,429(13.8)$ & $17,126(19.8)$ & $34,303(12.0)$ \\
\hline \multicolumn{4}{|l|}{ T2D treatment, $n(\%)$} \\
\hline No antidiabetic medication & $68,681(18.4)$ & $12,178(14.1)$ & $56,503(19.7)$ \\
\hline NIAD monotherapy & $138,615(37.1)$ & $28,582(33.0)$ & $110,033(38.4)$ \\
\hline NIADs in combination & $86,508(23.2)$ & $18,557(21.4)$ & $67,951(23.7)$ \\
\hline Insulin \pm NIAD & $79,381(21.3)$ & $27,217(31.5)$ & $52,164(18.2)$ \\
\hline
\end{tabular}

BMI: body mass index; eGFR: estimated glomerular filtration rate; NIAD: noninsulin antidiabetic drug; SD: standard deviation; T2D: type 2 diabetes; UACR: urine albumin-to-creatinine ratio; HbAlc: glycated haemoglobin. *Secondary prevention: patients with established cardiovascular disease (ICD-10 codes for coronary heart disease, cerebrovascular disease, or peripheral arteriopathy). ${ }^{* *}$ Primary prevention: patients without any ICD-10 code for cardiovascular disease. ${ }^{\dagger}$ There were $12 \%$ of missing data in the registration of $\mathrm{HbAlc}$ and $10 \%$ in the registration of eGFR.

trial), canagliflozin (CANVAS trial), and dapagliflozin (DECLARE-TIMI 58 trial) (Supplementary Table 1) [15-17].

2.4. Study Variables. The following variables from the SIDIAP database at the end of December 2016 were analysed: age and gender; duration of T2D; the most recent value (closest to 31 December 2016) of glycated haemoglobin (HbA1c); and presence of risk factors, including hypertension (ICD-10 codes I10 and I15, or systolic blood pressure $(\mathrm{SBP}) \geq 140$ and/or diastolic blood pressure $(\mathrm{DBP}) \geq 90 \mathrm{mmHg}$, or use of antihypertensive medications), dyslipidemia (ICD-10 codes E780 to E785, or LDL cholesterol (LDLc) $\geq 160 \mathrm{mg} / \mathrm{dl}$, or use of lipid-lowering drugs), smoking status, body mass index (BMI), estimated glomerular filtration rate (eGFR) using the Chronic Kidney Disease Epidemiology Collaboration (CKD-EPI) equation, albumin-to-creatinine ratio (UACR), and history of CVD (stroke, peripheral artery disease, ischaemic heart disease, and heart failure) (Supplementary Table 2). Noninsulin antidiabetic drugs (NIADs) and insulin-active electronic prescriptions on the index date were also considered.

2.5. Statistical Analysis. The eligibility was determined by dividing the number of patients fulfilling each of the CVOTs key inclusion and exclusion criteria by the total T2D regis- tered population. Data were summarised as mean (standard deviation (SD)) or $n(\%)$.

\section{Results}

A total of 373,185 patients with T2D were registered in the SIDIAP database as of 31 December 2016 (Table 1). The mean age was $70.1 \pm 12.3$ years, and $54.9 \%$ were male. The mean T2D duration was $9.3 \pm 6.2$ years, and the mean $\mathrm{HbA} 1 \mathrm{c}$ was $7.12 \% \pm 1.32$. More than half $(59 \%)$ of the patients had good glycaemic control (HbA1c $\leq 7 \%)$, about one-third $(28 \%)$ had chronic kidney disease (eGFR $<60 \mathrm{ml} / \mathrm{min} / 1.73 \mathrm{~m}^{2}$ ), and $4 \%$ had severe renal insufficiency $\left(\mathrm{eGFR}<30 \mathrm{ml} / \mathrm{min} / 1.73 \mathrm{~m}^{2}\right)$. Overall, $77 \%$ of patients $(n=286,651)$ did not have any ICD-10 code of established CVD recorded, thus considered primary prevention cases; $23 \%(n=86,534)$ had an ICD-10 code of an established CVD, so they were considered secondary prevention cases. Patients with established CVD were more often men, older, and with a longer T2D duration and had more CV risk factors (Table 1).

Applying the EMPA-REG OUTCOME eligibility criteria, only $8.2 \%(n=30,559)$ of the patients included in the SIDIAP database would have qualified for entry in the trial (Table 2$)$, while $29.6 \%(n=110,551)$ could have 
TABLE 2: Eligibility criteria for the EMPA-REG OUTCOME (empagliflozin) trial and number of patients in the SIDIAP database that would have met criteria for enrolment.

\begin{tabular}{lc}
\hline Eligibility criteria in EMPA-REG OUTCOME trial & Potentially eligible patients from the SIDIAP database $(N=373,185)$ \\
\hline Inclusion criteria & $n(\%)$ \\
Age $\geq 18$ years & $373,185(100)$ \\
Preexisting CV event: CHD, angina, MI, stroke, and PAD & $86,534(23.2)$ \\
HbAlc level $7.0 \%-\leq 10.0 \%$ & $33,270(8.9)$ \\
Main exclusion criteria & $n(\%)$ \\
eGFR $<30 \mathrm{ml} / \mathrm{min} / 1.73 \mathrm{~m}^{2}$ & $2,488(0.7)$ \\
BMI $>45 \mathrm{~kg} / \mathrm{m}^{2}$ & $223(0.06)$ \\
Total eligible, $n(\%)$ & $\mathbf{3 0 , 5 5 9 ( 8 . 2 )}$ \\
\hline
\end{tabular}

CHD: coronary heart disease; CV: cardiovascular; eGFR: estimated glomerular filtration rate; MI: myocardial infarction; PAD: peripheral artery disease; HbAlc: glycated haemoglobin; BMI: body mass index. All the percentages refer to the proportion from the total number of eligible subjects of the SIDIAP database $(n=373,185)$.

TABLE 3: Eligibility criteria for the CANVAS program (canagliflozin) and number of patients in the SIDIAP database that would have met criteria for enrolment.

\begin{tabular}{|c|c|c|}
\hline \multirow{2}{*}{$\begin{array}{l}\text { Eligibility criteria in the CANVAS program } \\
\text { Inclusion criteria }\end{array}$} & \multicolumn{2}{|c|}{$\begin{array}{l}\text { Potentially eligible patients from the SIDIAP } \\
\text { database }(n=373,185)\end{array}$} \\
\hline & In $\mathrm{PP}, n(\%)$ & In SP, $n(\%)$ \\
\hline \multicolumn{3}{|l|}{ Age: } \\
\hline$\geq 50$ years in $\mathrm{PP}$ & $349,896(93.8)$ & - \\
\hline$\geq 30$ years in $\mathrm{SP}$ & - & $372,764(99.9)$ \\
\hline \multicolumn{3}{|l|}{ Primary prevention cohort } \\
\hline \multicolumn{3}{|l|}{$\begin{array}{l}\quad \geq 50 \text { years and } \geq 2 \text { CVRF: } \\
\text { (i) T2D duration } \geq 10 \text { years }\end{array}$} \\
\hline \multicolumn{3}{|l|}{$\begin{array}{l}\text { (iv) Micro- or macroalbuminuria } \\
\text { (v) HDLc }-<39 \mathrm{mg} / \mathrm{dl}(1 \mathrm{mmol} / \mathrm{l})\end{array}$} \\
\hline \multicolumn{3}{|l|}{ Secondary prevention cohort } \\
\hline$\geq 30$ years and history of $\mathrm{CV}$ events ( $\mathrm{CHD}$, angina, $\mathrm{MI}$, stroke, and $\mathrm{PAD}$ ) & - & $86,531(23.2)$ \\
\hline HbA1c: $7.0 \%-10.5 \%$ & $83,537(22.4)$ & $34,320(9.2)$ \\
\hline Main exclusion criteria & In $\mathrm{PP}, n(\%)$ & In SP, $n(\%)$ \\
\hline $\mathrm{eGFR}<30 \mathrm{ml} / \mathrm{min} / 1.73 \mathrm{~m}^{2}$ & $4731(1.27)$ & $2575(0.69)$ \\
\hline Total eligible in PP and SP, $n(\%)$ & $78,806(21.1)$ & $31,745(8.5)$ \\
\hline Total eligible, $n(\%)$ & \multicolumn{2}{|c|}{$110,551(29.6)$} \\
\hline
\end{tabular}

CHD: coronary heart disease; CV: cardiovascular; CVRF: cardiovascular risk factors; eGFR: estimated glomerular filtration rate; MI: myocardial infarction; PP: primary cardiovascular prevention; SP: secondary cardiovascular prevention; PAD: peripheral artery disease; T2D: type 2 diabetes; HbAlc: glycated haemoglobin; HDLc: high-density lipoprotein cholesterol. All the percentages refer to the proportion from the total number of eligible subjects of the SIDIAP database $(n=373,185)$.

been enrolled in the CANVAS program (Table 3 ) and $38 \%$ $(n=141,653)$ would have been eligible for the DECLARETIMI 58 trial (Table 4). In Figure 1, these results are shown in comparison to those reported by two studies conducted in the US and Northern Europe [18, 19].

Compared with the characteristics of the patients enrolled in the three studied CVOTs (Table 5), the main limiting factors for inclusion would have been the absence of a history of CVD and the value of HbA1c. Specifically, only $23 \%$ of patients in our T2D population had a preexisting CVD and only $41 \%$ had $\mathrm{HbA} 1 \mathrm{c}$ values $\geq 7 \%$. Finally, chronic renal failure (present in $28 \%$ of our T2D patients) was a restrictive criterion in DECLARE, while in EMPAREG and
CANVAS, only patients with severe renal failure $\left(\mathrm{eGFR}<30 \mathrm{ml} / \mathrm{min} / 1.73 \mathrm{~m}^{2}\right.$ ) were excluded ( $4 \%$ in SIDIAP).

\section{Discussion}

In this population-based study using routine clinical data, we estimated the proportion of patients with T2D that would have been eligible for inclusion in the main CVOTs regarding the use of SGLT2i in our environment (Catalonia, Spain). We found that the DECLARE-TIMI 58 CVOT trial had the highest representativeness, covering $38 \%$ of the T2D patients in our general T2D population, which is in line with previously reported studies conducted in Northern Europe and the US 
TABLE 4: Eligibility criteria for the DECLARE-TIMI 58 trial (dapagliflozin) and number of patients in the SIDIAP database that would have met criteria for enrolment.

\begin{tabular}{|c|c|c|}
\hline \multirow{2}{*}{$\begin{array}{l}\text { Eligibility in the DELCLARE-TIMI } 58 \text { trial } \\
\text { Inclusion criteria }\end{array}$} & \multicolumn{2}{|c|}{$\begin{array}{l}\text { Potentially eligible patients from the SIDIAP } \\
\text { database }(n=373,185)\end{array}$} \\
\hline & In PP, $n(\%)$ & In SP, $n(\%)$ \\
\hline Age: & & \\
\hline (i) In PP: $\geq 55$ years; $\geq 60$ in women & $175,092(46.9) ; 140,941(37.8)$ & - \\
\hline (ii) In SP: $\geq 40$ & - & $368,963(98.9)$ \\
\hline \multicolumn{3}{|l|}{ Primary prevention cohort } \\
\hline $\begin{array}{l}\quad \geq 55 \text { years ( } \geq 60 \text { in women) and } \geq 1 \text { CVRF: } \\
\text { (i) Dyslipidemia } \\
\text { (ii) Hypertension } \\
\text { (iii) Current smoker }\end{array}$ & $289,126(77.5)$ & - \\
\hline \multicolumn{3}{|l|}{ Secondary prevention cohort } \\
\hline$\geq 40$ years and history of $\mathrm{CV}$ events (CHD, angina, $\mathrm{MI}$, stroke, and PAD) & - & $86,468(23.2)$ \\
\hline HbAlc $6.5 \%-<12 \%$ & $165,777(44.4)$ & $50,872(13.6)$ \\
\hline Main exclusion criteria & In PP & In SP \\
\hline $\mathrm{eGFR}<60 \mathrm{ml} / \mathrm{min} / 1.73 \mathrm{~m}^{2}$ & $53,851(14.4)$ & $21,145(5.7)$ \\
\hline Total eligible in PP and SP, $n(\%)$ & $111,926(30)$ & $29,727(7.9)$ \\
\hline Total eligible, $n(\%)$ & \multicolumn{2}{|c|}{$141,653(38 \%)$} \\
\hline
\end{tabular}

CHD: coronary heart disease; CV: cardiovascular; CVRF: cardiovascular risk factors; eGFR: estimated glomerular filtration rate; MI: myocardial infarction; PAD: peripheral artery disease; PP: primary cardiovascular prevention; SP: secondary cardiovascular prevention; HbAlc: glycated haemoglobin. All the percentages refer to the proportion from the total number of eligible subjects of the SIDIAP database $(n=373,185)$.

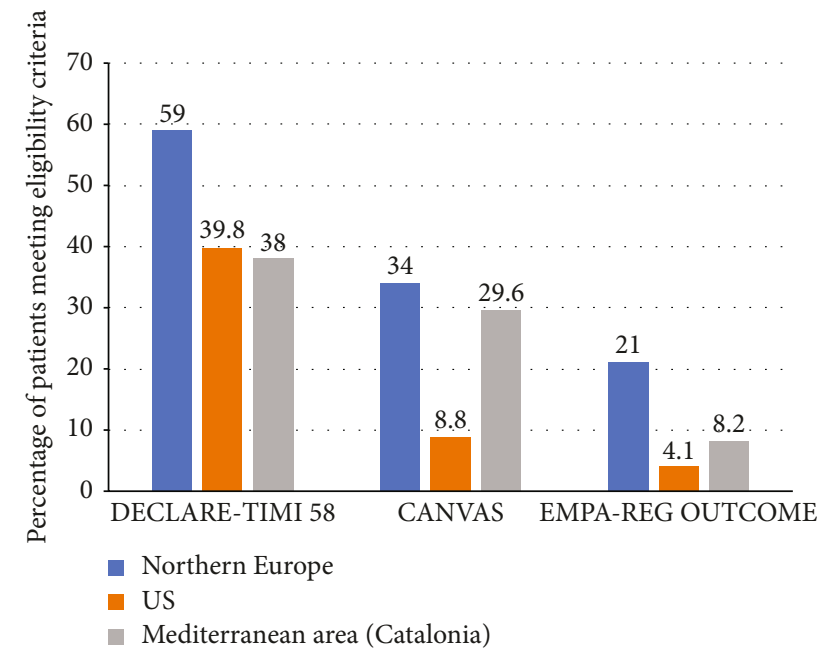

FIGURE 1: Graphical representation of the representativeness of patients in SGLT2i CVOTs when compared to the general type 2 diabetes population from four European countries, the US, and the present Mediterranean population.

$[13,17,25]$. This result is not surprising since the DECLARETIMI 58 CVOT trial included similar proportions of patients in primary $\mathrm{CV}$ prevention and secondary $\mathrm{CV}$ prevention (59\% and $41 \%$, respectively) [17], which ensures the highest representativeness. The CVOT with the lowest generalisability was the EMPA-REG OUTCOME trial because it included only secondary prevention patients [15]. However, if we only take into account patients with cardiovascular disease as eligible to enter into the trials, the resulting proportion would have been similar among the three trials: $8.2 \%$ for the
EMPAREG, $8.5 \%$ for the CANVAS program, and $7.9 \%$ for the DECLARE trial. Indeed, the main differences in the design of the different CVOT trials that we included were the enrolment of patients at high $\mathrm{CV}$ risk at baseline: EMPA-REG OUTCOME included only patients with T2D and established CV disease (i.e., secondary prevention), while both the CANVAS and the DECLARE-TIMI 58 also included primary prevention patients. In our real-world T2D population, the main limiting factors for inclusion in the different CVOTs analysed were the absence of a history of CV disease (present in the $23 \%$ of the patients) and glycaemic control: only $41 \%$ of the patients had a $\mathrm{HbA} 1 \mathrm{c} \geq 7 \%$ (entry criteria for the EMPA-REG OUTCOME and CANVAS trials), and $65.3 \%$ were above $6.5 \%$ threshold (entry criterion in the DECLARE-TIMI 58 study).

Concern regarding poor external validity (i.e., generalisability) of RCTs is largely known and has implications for the use (or underuse) of treatments in routine clinical practice $[14,26]$. This topic has also been addressed in the past regarding RCTs in the field of diabetes. For instance, in a previous literature review, the external validity of large trials assessing the impact of glycaemic control on CVD in patients with T2D was reported as limited when applied to a T2D population-based cohort [27]. Another recent study evaluated the population representativeness of 1691 registered T2D trials [28] and found that in $51.4 \%$ of cases (and $53.1 \%$ of phase 2 and 3 interventional trials), the population representativeness was $<5 \%$. Of note, and in line with our results, the eligibility criterion that had the largest effect on the population representativeness was HbAlc [28]. Finally, the study showed that the greater the number of eligibility criteria, the lower the representativeness was, and the authors concluded that the low representativeness of T2D trials could 
TABLE 5: Summary of characteristics of patients enrolled in SGLT2i CVOTs and of patients from the SIDIAP database that would have met the corresponding criteria.

\begin{tabular}{|c|c|c|c|c|}
\hline Characteristics & $\begin{array}{l}\text { EMPA-REG } \\
\text { OUTCOME }\end{array}$ & CANVAS & DECLARE-TIMI 58 & General population (SIDIAP database) \\
\hline Drug & Empagliflozin & Canagliflozin & Dapagliflozin & Empagliflozin/canagliflozin/dapagliflozin \\
\hline Participants, $n$ & 7,020 & 10,142 & 17,160 & 373,185 \\
\hline Male (\%) & 71.5 & 64.2 & 62.6 & 54.9 \\
\hline Age (years), mean (SD) & $63.1(8.7)$ & $63.3(8.3)$ & $63.9(6.8)$ & $70.1(12.3)$ \\
\hline $\begin{array}{l}\text { Patients with established } \\
\text { CVD, } n(\%)\end{array}$ & $7,020(>99)$ & $6,656(66)$ & $6,974(41)$ & $\begin{array}{l}\text { EMPA-REG OUTCOME criteria: } \\
\text { 86,534 (23.2) } \\
\text { CANVAS criteria: } 86,531(23.2) \\
\text { DECLARE-TIMI } 58 \text { criteria: } 86,468(23.2)\end{array}$ \\
\hline CVRFs, $n(\%)$ & - & $3486(34)$ & $10,186(59)$ & $\begin{array}{c}\text { CANVAS criteria: } 189,969(50.9) \\
\text { DECLARE-TIMI } 58 \text { criteria: } 289,126(77.5)\end{array}$ \\
\hline HbAlc (\%), mean (SD) & $8.1(0.8)$ & $8.2(0.9)$ & $8.3(1.2)$ & $7.12(1.32)$ \\
\hline \multicolumn{5}{|l|}{$\mathrm{eGFR}\left(\mathrm{ml} / \mathrm{min} / 1.73 \mathrm{~m}^{2}\right), n(\%)$} \\
\hline$\geq 60$ & $5,199(74.1)$ & $8,114(79.9)$ & $15,959(92.6)$ & $241,958(72)$ \\
\hline$<60-30$ & $1,819(25.9)$ & $2,028(20.1)$ & $1,201(7.4)$ & $80,978(24)$ \\
\hline$<30$ & 0 & 0 & 0 & $13,262(4)$ \\
\hline
\end{tabular}

CVD: cardiovascular disease; CVRF: cardiovascular risk factors; eGFR: estimated glomerular filtration rate; SD: standard deviation; CVOTs: cardiovascular outcome trials.

be attributed to safety concerns when designing the study, which may lead to overly restrictive eligibility criteria to prevent adverse events [28].

Chronic renal failure was a restrictive criterion in DECLARE, while EMPA-REG OUTCOME and CANVAS trials only excluded patients with severe renal insufficiency $\left(<30 \mathrm{ml} / \mathrm{min} / 1.73 \mathrm{~m}^{2}\right)$, present in $4 \%$ of our population. However, renal benefits of SGLT2i have been shown in different studies and one meta-analysis [29], particularly in the EMPA-REG OUTCOME and CANVAS trials [15, 16, 3032], although these results were from secondary outcomes. New trials are ongoing with the primary renal endpoint (kidney outcome trials) focusing on patients with T2D and established chronic kidney disease (CKD) $[33,34]$. The latest recommendations from the 2018 ADA/EASD Consensus Guidelines suggest to consider the use of a SGLT2 $\mathrm{i}$ in patients with T2D and CKD [35]. In fact, the FDA has recently modified this limitation for patients with moderate renal impairment (i.e., eGFR45 $60 \mathrm{ml} / \mathrm{min} / 1.73 \mathrm{~m}^{2}$ ) for dapagliflozin, as previously done with canagliflozin $100 \mathrm{mg}$ and empagliflozin $10 \mathrm{mg}$ [36]. In summary, in the US, SGLT2i are still not recommended when eGFR is less than $45 \mathrm{ml} / \mathrm{min} / 1.73 \mathrm{~m}^{2}$ and remain contraindicated in patients with severe renal impairment $\left(\mathrm{eGFR}<30 \mathrm{ml} / \mathrm{min} / 1.73 \mathrm{~m}^{2}\right.$ ), end-stage renal disease, or on dialysis [36]. Conversely, the EMA has not yet modified the general restriction of eGFR $<60 \mathrm{ml} / \mathrm{min} / 1.73 \mathrm{~m}^{2}$. However, it is likely that recommendations by drug agencies for SGLT2i in CKD will change in the future after the publication of the results from the ongoing kidney outcome trials (CREDENCE, DAPA-CKD, and EMPAGLIFLOZIN RENAL) [37]. In addition, recent results of the CREDENCE trial showed that the risk of kidney failure and renal or $\mathrm{CV}$ mortality was 30\% lower in the group of patients receiving canagliflozin compared to placebo (HR for the primary composite outcome of end-stage kidney disease, doubling of serum creatinine, or renal or $\mathrm{CV}$ death $=0.70 ; 95 \%$ CI, 0.59-0.82) [38]. All the patients included in this trial had an eGFR between 30 and $90 \mathrm{ml} / \mathrm{min} / 1.73 \mathrm{~m}^{2}$ and albuminuria, and in a subgroup analysis, the renal benefit of canagliflozin was higher among patients with eGFR between 45 and $60 \mathrm{ml} / \mathrm{min} / 1.73 \mathrm{~m}^{2}$ (HR 0.52; 95\% CI, 0.38-0.72) [38].

Indeed, a recent systematic review and trial-level metaanalysis of SGLT2i CVOT trials concludes that SGLT2 $\mathrm{i}$ reduce the risk of worsening eGFR in a broad spectrum of T2D patients [39]. Considering the facts mentioned above, if SGLT2i were allowed to be prescribed to patients with $\mathrm{eGFR}>30 \mathrm{ml} / \mathrm{min} / 1.73 \mathrm{~m}^{2}$, the proportion of patients eligible for inclusion in the DECLARE TIMI 58 in the SIDIAP database would have increased.

The results of the present study show that the patterns of external validity of SGLT2i CVOTs were limited, in line with the studies conducted in Northern Europe or the US $[18,19]$. However, the proportion of eligible patients would have been less in our population than in Northern Europe for all three trials but higher than that in the US for the CANVAS and EMPAREG studies as shown in Figure 1. This could be explained by differences in the prevalence of CVD between regions and countries. Indeed, the prevalence of CVD is higher in countries from the north of Europe than from the south of Europe [21], but it is also probable that differences in the prevalence of specific CV risk factors by country and region have impacted our results. The low eligibility for CANVAS and EMPA-REG OUTCOME in the US study is striking, although the percentage of patients having CVD was similar to ours (23.7 and $23 \%$, respectively). One explanation for this discordance could be related to the different methodologies used in the US study, which is an estimation 
based on data from two NHANES surveys. In this study, data from 20,293 volunteer subjects, 2,395 of whom had T2D, were extrapolated to $23,941,512$ persons having T2D in the US [19]. For instance, these patients were younger than in the other databases: 59 years old in the US, 68 years in the European study, and 70 years in our study. As a result, the prevalence of $\mathrm{CV}$ risk factors was lower in our database (13.6\% in the US vs. $28 \%$ ). Conversely, the prevalence of CVD in the European study (43.7\% in Germany, 34.8\% in The Netherlands, $31.4 \%$ in Sweden, and $25.1 \%$ in Norway) was higher in our database (23\%) and produced higher percentages of inclusion in the CANVAS and EMPA-REG OUTCOME trials [18].

On the other hand, the applicability of SGLT2i is certainly much wider than the strict entry criteria of CVOTs, as shown in several of studies and subanalyses reporting the additional benefits of SGLT2i, particularly in chronic kidney disease and heart failure [40]. For instance, real-world evidence (RWE) studies have also confirmed reductions in mortality (43\% to $49 \%$ ) and hospitalization for heart failure ( $40 \%$ to $51 \%$ ) in hundreds of thousands of patients [40]. So far, the main RWE studies published are CVD-Real, CVD-Real 2, EASEL, and EMPRISE; these studies compared SGLT2i with other antidiabetic drugs, especially against DPP-4 inhibitors (CVD-Real 2 and EMPRISE). However, our study aimed at determining how many patients from our Mediterranean database would be eligible to enter in each CVOT, but not the applicability of SGLT2i in the whole diabetic population. Thus, even though we found a low percentage of patients that would have been enrolled in each of the cardiovascular outcome trials, we should not dismiss the additional benefits of using SGLT2i in the whole diabetic population in terms of heart failure benefit. Hence, our results should be taken with caution and should not drive the decision to prescribe or not an SGLT2i in routine practice without considering all clinical aspects and patient preferences. Finally, we should point out that, at the time of deciding whether to prescribe or not an SGLT2i, clinicians should also refer to the latest available evidence and updated guidelines for T2DM management [35, 41]. In this line, the 2018 Consensus Report of the American Diabetes Association (ADA) and the European Association for the Study of Diabetes (EASD) state that, in the presence of cardiovascular disease, empagliflozin or canagliflozin should be recommended if HbAlc levels are above target, usually above 7\% [35]. Moreover, the recently published 2019 ESC Guidelines in collaboration with the EASD recommend the use of SGLT2i in case of established cardiovascular disease or in subjects at high or very high CV risk, without establishing any specific threshold for HbA1c [41].

This study has some limitations. The main limitations derive from its retrospective observational nature, which are common to all similarly designed studies using realworld databases. For instance, $12 \%$ and $10 \%$ of patients had no available $\mathrm{HbA1c}$ or eGFR values during the evaluated year, respectively. On the other hand, the strength of the present study is that it involves real-world data from a Med- iterranean region where the prevalence of $\mathrm{CV}$ risk factors and CVD in patients with T2D is expected to be different from that in Northern Europe or the US [21].

\section{Conclusions}

This study shows that there are considerable differences in the external validity of the different CVOTs of SGLT2i when applying the same eligibility criteria to the T2D population of Catalonia. The DECLARE-TIMI 58 CVOT was the most generalisable, while the EMPA-REG OUTCOME and CANVAS trials were much less representative of real-world T2D patients. However, the clinical applicability of SGLT2i in routine practice goes beyond the strict inclusion criteria of CVOTs and it is important to consider all patient-centered aspects before decision-making in T2D management.

\section{Data Availability}

The data used to support the findings of this study are available from the corresponding authors upon request.

\section{Disclosure}

The funding source had no role in the design and conduct of the study; collection, management, analysis, and interpretation of the data; or preparation, review, or approval of the manuscript.

\section{Conflicts of Interest}

M.M.-C. has received advisory honorarium from AstraZeneca, Bayer, Boehringer Ingelheim, GSK, Lilly, MSD, Novartis, Novo Nordisk, and Sanofi; he has received speaker honorarium from Astra-Zeneca, Bayer, Boehringer Ingelheim, GSK, Lilly, Menarini, MSD, Novartis, Novo Nordisk, and Sanofi; he has received research grants to the institution from Astra-Zeneca, GSK, Lilly, MSD, Novartis, Novo Nordisk, and Sanofi. J.F.-N. has received advisory and/or speaking fees from Astra-Zeneca, Ascensia, Boehringer Ingelheim, GSK, Lilly, MSD, Novartis, Novo Nordisk, and Sanofi; he has received research grants to the institution from Astra-Zeneca, GSK, Lilly, MSD, Novartis, Novo Nordisk, Sanofi, and Boehringer. D.M. has received advisory and/or speaking fees from Astra-Zeneca, Ascensia, Boehringer Ingelheim, GSK, Lilly, MSD, Novartis, Novo Nordisk, and Sanofi; he has received research grants to the institution from Astra-Zeneca, GSK, Lilly, MSD, Novartis, Novo Nordisk, Sanofi, and Boehringer. S.C., J.R., B.V., and M.G. have no conflicts of interest to declare.

\section{Authors' Contributions}

Silvia Canivell and Manel Mata-Cases contributed equally to this work.

\section{Acknowledgments}

The authors acknowledge Amanda Prowse (Lochside Medical Communications Ltd.) for providing support in editing 
the paper. This study was funded by the Fundació Institut Universitari per a la recerca a l'Atenció Primària de Salut Jordi Gol i Gurina (IDIAPJGol). CIBER of Diabetes and Associated Metabolic Diseases (CIBERDEM) is an initiative from Instituto de Salud Carlos III, Madrid, Spain.

\section{Supplementary Materials}

Supplementary Table 1: eligibility criteria of the three studied CVOTs completed for SGLT2i in T2D patients. Supplementary Table 2: ICD-10 codes for cardiovascular diseases considered in the study. (Supplementary Materials)

\section{References}

[1] Emerging Risk Factors Collaboration, N. Sarwar, P. Gao et al., "Diabetes mellitus, fasting blood glucose concentration, and risk of vascular disease: a collaborative meta-analysis of 102 prospective studies," The Lancet, vol. 375, no. 9733, pp. 2215-2222, 2010.

[2] I. M. Stratton, A. I. Adler, H. A. Neil et al., "Association of glycaemia with macrovascular and microvascular complications of type 2 diabetes (UKPDS 35): prospective observational study," BMJ, vol. 321, no. 7258, pp. 405-412, 2000.

[3] ADVANCE Collaborative Group, A. Patel, S. MacMahon et al., "Intensive blood glucose control and vascular outcomes in patients with type 2 diabetes," New England Journal of Medicine, vol. 358, no. 24, pp. 2560-2572, 2008.

[4] N. Marx, J. Rosenstock, S. E. Kahn et al., "Design and baseline characteristics of the CARdiovascular Outcome Trial of LINAgliptin versus glimepiride in type 2 diabetes (CAROLINA ${ }^{\circledR}$ )," Diabetes and Vascular Disease Research, vol. 12, no. 3, pp. 164-174, 2015.

[5] M. H. Secrest, J. A. Udell, and K. B. Filion, "The cardiovascular safety trials of DPP-4 inhibitors, GLP-1 agonists, and SGLT2 inhibitors," Trends in Cardiovascular Medicine, vol. 27, no. 3, pp. 194-202, 2017.

[6] M. A. Nauck, J. J. Meier, M. A. Cavender, M. Abd el Aziz, and D. J. Drucker, "Cardiovascular actions and clinical outcomes with glucagon-like peptide-1 receptor agonists and dipeptidyl peptidase-4 inhibitors," Circulation, vol. 136, no. 9, pp. 849870, 2017.

[7] E. Mannucci, M. Monami, C. Lamanna, F. Gori, and N. Marchionni, "Prevention of cardiovascular disease through glycemic control in type 2 diabetes: a meta-analysis of randomized clinical trials," Nutrition, metabolism, and cardiovascular diseases : NMCD, vol. 19, no. 9, pp. 604-612, 2009.

[8] US Food and Drug Administration (FDA), "Guidance for Industry. Diabetes mellitus - evaluating cardiovascular risk in new antidiabetic therapies to treat type 2 diabetes. FDA website," March, 2019, http://www.fda.gov/downloads/ Drugs/.../Guidances/ucm071627.pdf.

[9] European Medicines Agency, (EMA), "Guideline on clinical investigation of medicinal products in the treatment or prevention of diabetes mellitus," March 2019, https://www.ema .europa.eu/en/documents/scientific-guideline/draft-guidelineclinical-investigation-medicinal-products-treatmentprevention-diabetes-mellitus_en.pdf.

[10] M. J. B. van Baar, C. C. van Ruiten, M. H. A. Muskiet, L. van Bloemendaal, R. G. IJzerman, and D. H. van Raalte, "SGLT2 inhibitors in combination therapy: from mechanisms to clini- cal considerations in type 2 diabetes management," Diabetes Care, vol. 41, no. 8, pp. 1543-1556, 2018.

[11] T. A. Zelniker, S. D. Wiviott, I. Raz et al., "SGLT2 inhibitors for primary and secondary prevention of cardiovascular and renal outcomes in type 2 diabetes: a systematic review and metaanalysis of cardiovascular outcome trials," The Lancet, vol. 393, no. 10166, pp. 31-39, 2019.

[12] J. H. Y. Wu, C. Foote, J. Blomster et al., "Effects of sodiumglucose cotransporter-2 inhibitors on cardiovascular events, death, and major safety outcomes in adults with type 2 diabetes: a systematic review and meta-analysis," The Lancet Diabetes \& Endocrinology, vol. 4, no. 5, pp. 411-419, 2016.

[13] K. I. Birkeland, M. E. Jørgensen, B. Carstensen et al., "Cardiovascular mortality and morbidity in patients with type 2 diabetes following initiation of sodium-glucose co-transporter-2 inhibitors versus other glucose-lowering drugs (CVD-REAL Nordic): a multinational observational analysis," The Lancet Diabetes \& Endocrinology, vol. 5, no. 9, pp. 709-717, 2017

[14] P. M. Rothwell, "External validity of randomised controlled trials: "To whom do the results of this trial apply?"," Lancet (London, England), vol. 365, no. 9453, pp. 82-93, 2005.

[15] B. Zinman, C. Wanner, J. M. Lachin et al., "Empagliflozin, cardiovascular outcomes, and mortality in type 2 diabetes," The New England Journal of Medicine, vol. 373, no. 22, pp. $2117-$ 2128, 2015.

[16] B. Neal, V. Perkovic, K. W. Mahaffey et al., "Canagliflozin and cardiovascular and renal events in type 2 diabetes," The New England Journal of Medicine, vol. 377, no. 7, pp. 644-657, 2017.

[17] S. D. Wiviott, I. Raz, M. P. Bonaca et al., "Dapagliflozin and cardiovascular outcomes in type 2 diabetes," The New England Journal of Medicine, vol. 380, no. 4, pp. 347-357, 2019.

[18] K. I. Birkeland, J. Bodegard, A. Norhammar et al., "How representative of a general type 2 diabetes population are patients included in cardiovascular outcome trials with SGLT2 inhibitors? A large European observational study," Diabetes, Obesity and Metabolism, vol. 21, no. 4, 2019.

[19] E. T. Wittbrodt, J. M. Eudicone, K. F. Bell, D. M. Enhoffer, K. Latham, and J. B. Green, "Eligibility varies among the 4 sodium-glucose cotransporter-2 inhibitor cardiovascular outcomes trials: implications for the general type 2 diabetes US population," The American Journal of Managed Care, vol. 24, 8 Suppl, pp. S138-S145, 2018.

[20] T. Tamayo, J. Rosenbauer, S. H. Wild et al., "Diabetes in Europe: an update," Diabetes Research and Clinical Practice, vol. 103, no. 2, pp. 206-217, 2014.

[21] T. R. Einarson, A. Acs, C. Ludwig, and U. H. Panton, "Prevalence of cardiovascular disease in type 2 diabetes: a systematic literature review of scientific evidence from across the world in 2007-2017," Cardiovascular Diabetology, vol. 17, no. 1, p. 83, 2018.

[22] M. Mata-Cases, D. Mauricio, J. Real, B. Bolíbar, and J. FranchNadal, “ ¿Se registra y clasifica correctamente la diabetes mellitus en atencion primaria? Estudio poblacional en Cataluña, España," Endocrinología y Nutrición, vol. 63, no. 9, pp. 440448, 2016

[23] M. Mata-Cases, J. Franch-Nadal, J. Real et al., "Therapeutic inertia in patients treated with two or more antidiabetics in primary care: factors predicting intensification of treatment," Diabetes, Obesity and Metabolism, vol. 20, no. 1, pp. 103112,2018 
[24] I. Vinagre, M. Mata-Cases, E. Hermosilla et al., "Control of glycemia and cardiovascular risk factors in patients with type 2 diabetes in primary care in Catalonia (Spain)," Diabetes Care, vol. 35, no. 4, pp. 774-779, 2012.

[25] S. V. Arnold, S. E. Inzucchi, F. Tang et al., "Real-world use and modeled impact of glucose-lowering therapies evaluated in recent cardiovascular outcomes trials: an $\mathrm{NCDR}^{\circledR}$ Research to Practice project," European Journal of Preventive Cardiology, vol. 24, no. 15, pp. 1637-1645, 2017.

[26] P. M. Rothwell, "Factors that can affect the external validity of randomised controlled trials," PLoS Clinical Trials, vol. 1, no. 1, p. e9, 2006.

[27] C. Saunders, C. D. Byrne, B. Guthrie et al., "External validity of randomized controlled trials of glycaemic control and vascular disease: how representative are participants?," Diabetic Medicine, vol. 30, no. 3, pp. 300-308, 2013.

[28] A. Sen, A. Goldstein, S. Chakrabarti et al., "The representativeness of eligible patients in type 2 diabetes trials: a case study using GIST 2.0," Journal of the American Medical Informatics Association, vol. 25, no. 3, pp. 239-247, 2017.

[29] S. Seidu, S. K. Kunutsor, X. Cos, S. Gillani, K. Khunti, and For and on behalf of Primary Care Diabetes Europe, "SGLT2 inhibitors and renal outcomes in type 2 diabetes with or without renal impairment: a systematic review and meta-analysis," Primary Care Diabetes, vol. 12, no. 3, pp. 265-283, 2018.

[30] V. Vallon and S. C. Thomson, "Diabetes mellitus: cardiovascular and renal benefits of SGLT2 inhibition: insights from CANVAS," Nature Reviews Nephrology, vol. 13, no. 9, pp. 517-518, 2017.

[31] C. Lo, T. Toyama, Y. Wang et al., "Insulin and glucoselowering agents for treating people with diabetes and chronic kidney disease," The Cochrane Database of Systematic Reviews, vol. 9, 2018.

[32] C. Wanner, S. E. Inzucchi, J. M. Lachin et al., "Empagliflozin and progression of kidney disease in type 2 diabetes," The New England Journal of Medicine, vol. 375, no. 4, pp. 323334, 2016.

[33] M. J. Jardine, K. W. Mahaffey, B. Neal et al., "The Canagliflozin and Renal Endpoints in Diabetes with Established Nephropathy Clinical Evaluation (CREDENCE) study rationale, design, and baseline characteristics," American Journal of Nephrology, vol. 46, no. 6, pp. 462-472, 2017.

[34] H. Jakher, T. I. Chang, M. Tan, and K. W. Mahaffey, "Canagliflozin review - safety and efficacy profile in patients with T2DM," Diabetes, Metabolic Syndrome and Obesity: Targets and Therapy, vol. Volume 12, pp. 209-215, 2019.

[35] M. J. Davies, D. A. D’Alessio, J. Fradkin et al., "Management of hyperglycemia in type 2 diabetes, 2018. A consensus report by the American Diabetes Association (ADA) and the European Association for the Study of Diabetes (EASD)," Diabetes Care, vol. 41, no. 12, pp. 2669-2701, 2018.

[36] M. E. Tucker, "FDA approves expanded uses for type 2 diabetes drugs. Medscape Medical News," 2019, March 2019, https://www.medscape.com/viewarticle/909713.

[37] R. Pecoits-Filho and V. Perkovic, "Are SGLT2 inhibitors ready for prime time for CKD?," Clinical Journal of the American Society of Nephrology, vol. 13, no. 2, pp. 318-320, 2018.

[38] V. Perkovic, D. de Zeeuw, K. W. Mahaffey et al., "Canagliflozin and renal outcomes in type 2 diabetes: results from the CANVAS program randomised clinical trials," The Lancet Diabetes and Endocrinology, vol. 6, no. 9, pp. 691-704, 2018.
[39] T. A. Zelniker, S. D. Wiviott, I. Raz et al., "Comparison of the effects of glucagon-like peptide receptor agonists and sodiumglucose Cotransporter 2 inhibitors for prevention of major adverse cardiovascular and renal outcomes in type 2 diabetes Mellitus," Circulation, vol. 139, no. 17, pp. 2022-2031, 2019.

[40] G. Schernthaner, H. Drexel, E. Moshkovich et al., "SGLT2 inhibitors in T2D and associated comorbidities - differentiating within the class," BMC Endocrine Disorders, vol. 19, no. 1, p. 64, 2019.

[41] F. Cosentino, P. J. Grant, V. Aboyans et al., "2019 ESC Guidelines on diabetes, pre-diabetes, and cardiovascular diseases developed in collaboration with the EASD," European Heart Journal, 2019. 


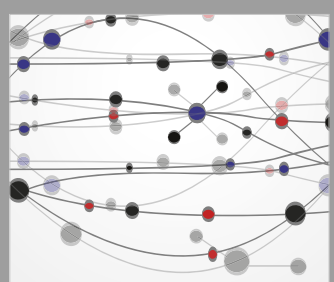

The Scientific World Journal
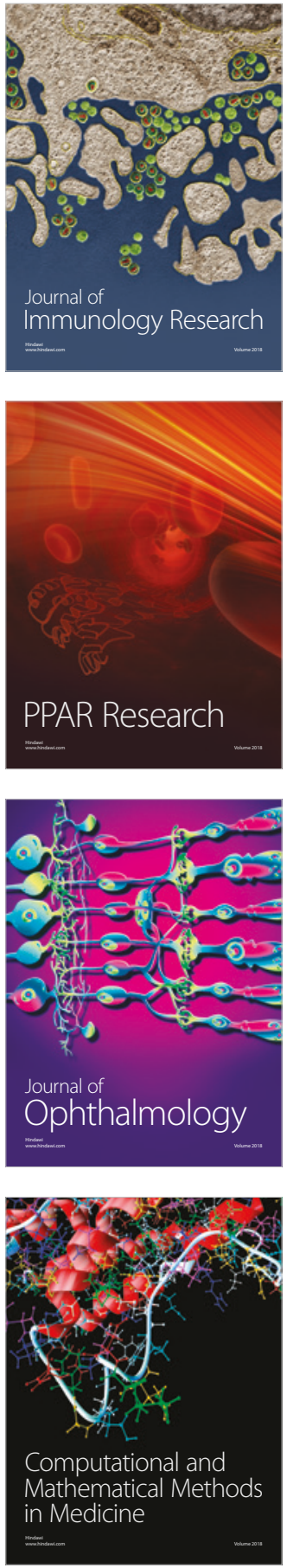

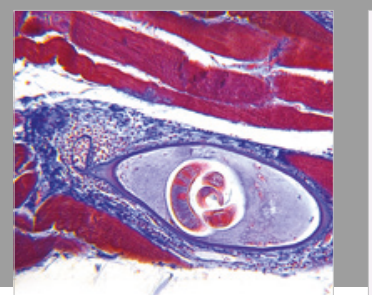

Gastroenterology Research and Practice

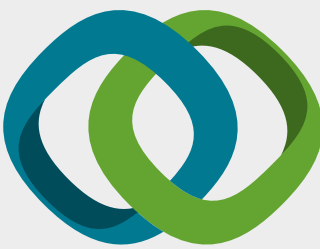

\section{Hindawi}

Submit your manuscripts at

www.hindawi.com
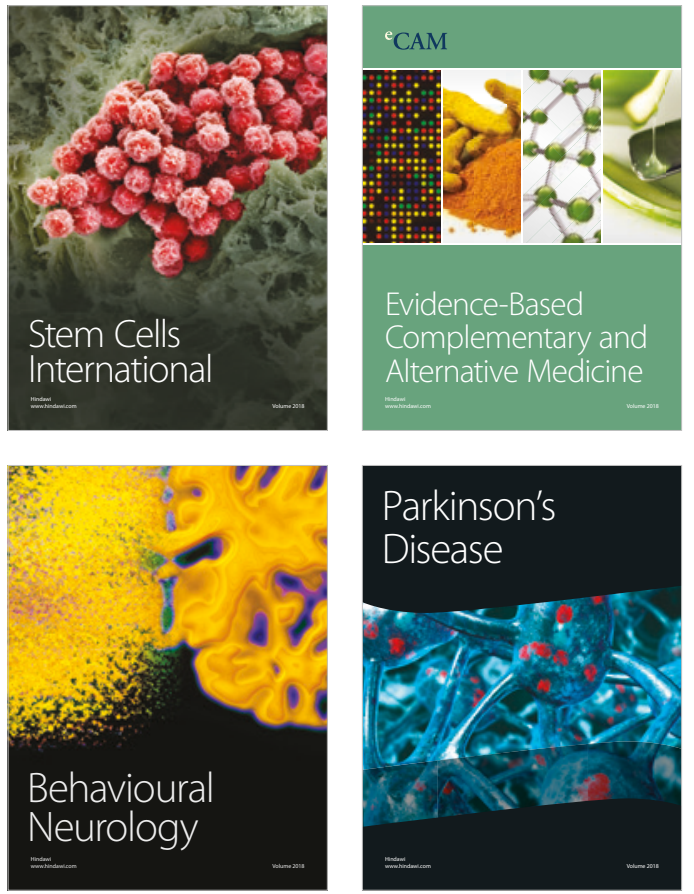

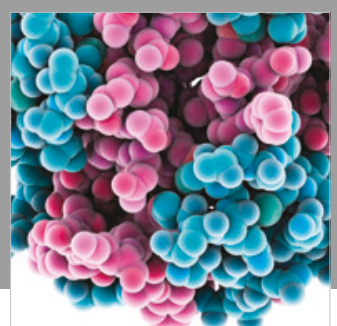

ournal of

Diabetes Research

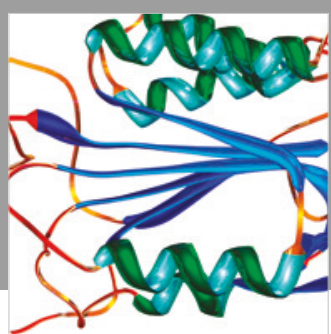

Disease Markers
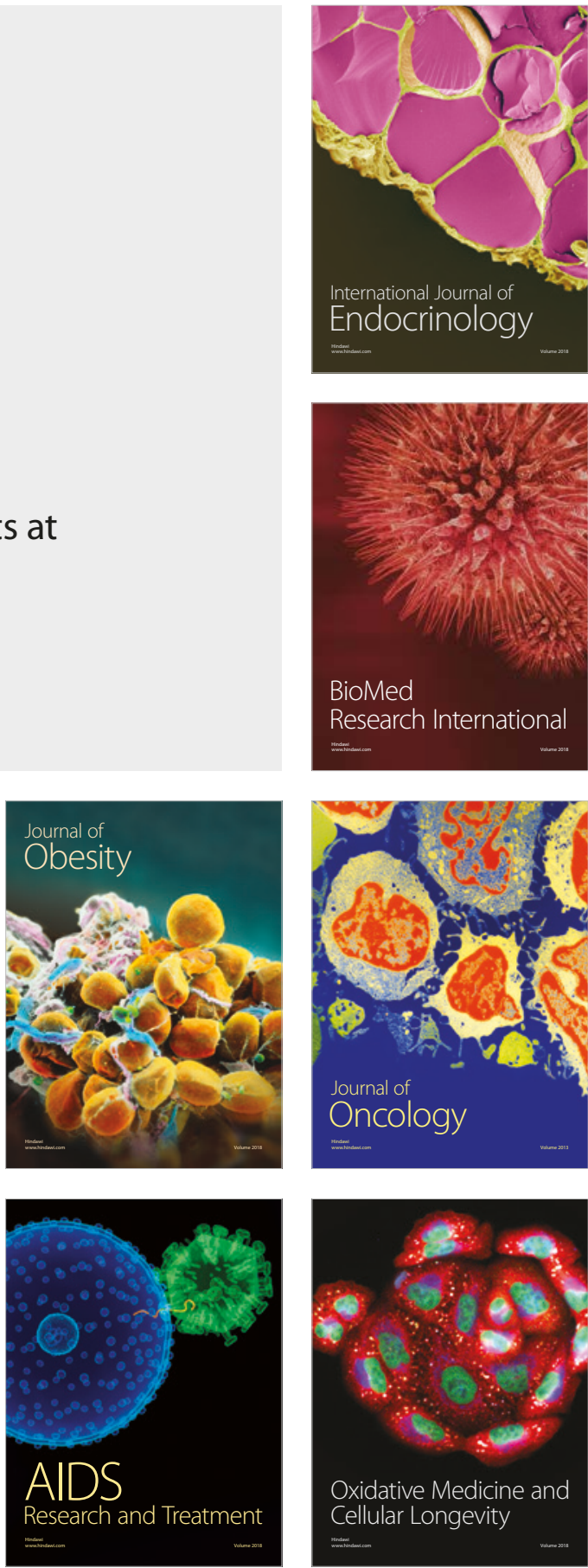